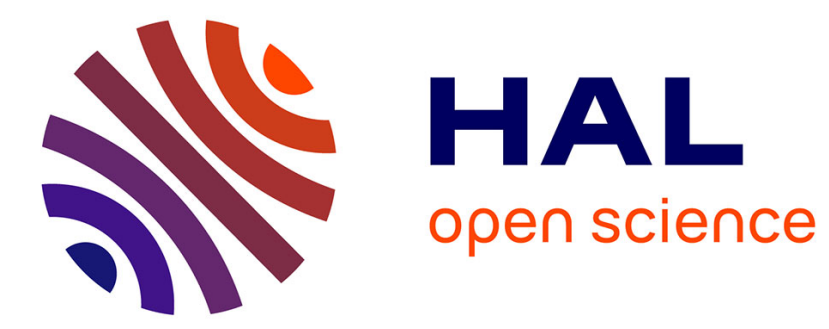

\title{
Effective noise reduction on pulsed laser excitation of modes in a high-Q photoacoustic resonator
}

\author{
A. Miklós, C. Brand, A. Winkler, P. Hess
}

\section{To cite this version:}

A. Miklós, C. Brand, A. Winkler, P. Hess. Effective noise reduction on pulsed laser excitation of modes in a high-Q photoacoustic resonator. Journal de Physique IV Proceedings, 1994, 04 (C7), pp.C7-781-C7-784. 10.1051/jp4:19947184 . jpa-00253246

\section{HAL Id: jpa-00253246 https://hal.science/jpa-00253246}

Submitted on 1 Jan 1994

HAL is a multi-disciplinary open access archive for the deposit and dissemination of scientific research documents, whether they are published or not. The documents may come from teaching and research institutions in France or abroad, or from public or private research centers.
L'archive ouverte pluridisciplinaire HAL, est destinée au dépôt et à la diffusion de documents scientifiques de niveau recherche, publiés ou non, émanant des établissements d'enseignement et de recherche français ou étrangers, des laboratoires publics ou privés. 


\title{
Effective noise reduction on pulsed laser excitation of modes in a high-Q photoacoustic resonator
}

\author{
A. Miklós(1),(2), C. Brand, A. Winkler and P. Hess \\ Institute of Physical Chemistry, University of Heidelberg, Im Newenheimer Feld 253, 69120 Heidelberg, \\ Germany
}

\begin{abstract}
An open photoacoustic detector system, consisting of a high $Q$ cylindrical resonator ( $Q$ factor in $N_{2} \approx 800$ ) and two adjoining variable acoustic filter systems, was tested for pulsed excitation of acoustical modes in a naphthalene/air gas mix by using a $308 \mathrm{~nm}$ excimer laser. The outside noise suppression and the signal-to-noise ratio were investigated in a normal laboratory environment in the flow-through mode. The acoustic and electric filter system combined with the averaging of the photoacoustic signal in the time domain suppresses the outside noise by $70 \mathrm{~dB}$. In this case the signal-to-noise ratio was $76 \mathrm{~dB}$.
\end{abstract}

\section{Introduction}

To reduce wall adsorption problems, photoacoustic (PA) gas detection with continuous gas flow would be favorable for in situ analysis. However, such a flow-through system is open from the acoustical point of view, thus difficulties are expected concerning the pick-up of outside noise. Although stationary noise signals may be suppressed by fitting acoustic filters at the openings of the resonant PA cell [1], sudden noise events, which are very common in the environment, will still disturb the PA detection considerably.

One possibility for the improvement of PA gas monitors is measurement in the time domain employing pulsed excitation in an open high-Q resonant PA cell. In this case the PA response will not be correlated to the outside noise. Therefore the application of the method of synchronized averaging of the responses will increase the signal-to-noise $(S / N)$ ratio very efficiently. Moreover, this method is also effective in suppressing pulsed noise signals originating from the environment.

Pulsed excitation followed by Fourier transformation (FT) of the time-resolved response signal has another advantage: it is not sensitive to a slow drift of the resonant frequency caused by temperature and pressure changes, because the analysis determinies not only the magnitude of the resonance peak but also its frequency. Therefore, it is possible to improve the $S / N$ ratio by simply using resonators with higher $Q$ values. In the case of continuously modulated excitation the synchronization of the modulation frequency to the actual resonance frequency of the PA cell is a serious problem. Common applications therefore use low-Q resonators in order to be independent of the fluctuations. Although the problem can be solved by applying an additional control loop for the light modulator [2], the system becomes more complicated compared to the pulsed one.

Only a few papers have been published on pulsed PA experiments [3, 4], and only one on the excitation of acoustic resonances by nanosecond laser pulses [4]. The aim of the present. work is to investigate the PA response of an open resonator system to the nanosecond pulses of a $308 \mathrm{~nm}$ excimer laser, to determine the noise performance of the system in a normal laboratory environment with and without gas flow, and to compare the results with the experimental data

(1) Present address: Wolfmahdenstrasse 70, 70563 Stuttgart, Germany

(2) Permanent address: Institute of Isotopes of the Hungarian Academy of Sciences, P.O. Box 77, 1525 Budapest, Hungary 


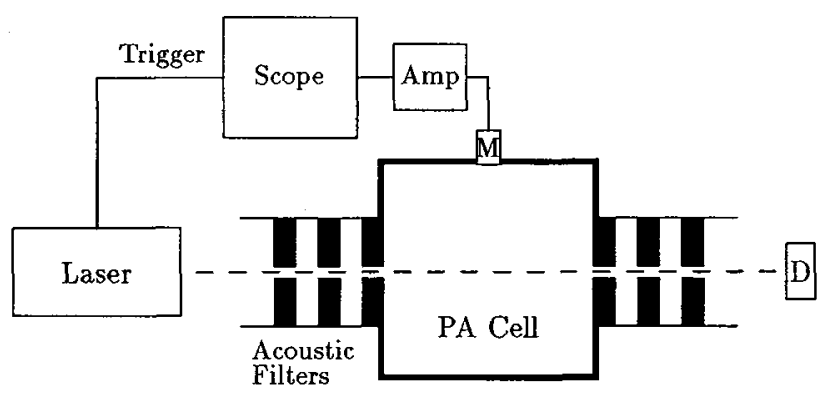

Figure 1: Scheme of the experimental setup, $\mathrm{M}=$ microphone, Amp = preamplifier and electric filters, $\mathrm{D}=$ light detector

of a closed resonator with windows at both ends of the acoustical filters.

\section{Experimental}

The PA detector system built for the present experiments (Fig. 1) is an improved modular version of an open PA detector design published earlier [1]. The inner cylindrical resonator is $5.0 \mathrm{~cm}$ in radius and $10.0 \mathrm{~cm}$ long. The $Q$ factor of the resonator is $\approx 500$ in air and $\approx 800 \mathrm{in} \mathrm{N}_{2}$. The laser beam is focused at the center of the cell. The distance of the laser beam to the cell axis can be changed. The microphone is positioned halfway along the cylinder surface. Five acoustic filter elements are mounted at each end of the cell. Their dimensions can be changed to suppress various spectral components of the outside noise [5]. In order to suppress noise in the range of the first radial mode of the resonator, the three solid $\lambda / 4$ filters had an inner radius of $3.0 \mathrm{~mm}$ and a length of $16.6 \mathrm{~mm}$, and the two acoustically optimized filter volumes in between had an inner radius of $24.7 \mathrm{~mm}$ and a length of $13.3 \mathrm{~mm}$. Many different arrangements can be realized by changing the corresponding modules.

The PA signal was measured by an electret microphone (Sennheiser KE4, sensitivity $\approx 35 \mathrm{mV} / \mathrm{Pa}$ ) coupled to a selective preamplifier. Further noise reduction was ensured by the bandpass filters of the preamplifier. The field-free frequency response of the entire microphoneamplifier circuit was was found to be linear in the range from 1 to $5 \mathrm{kHz}$, while frequencies below and above this passband were efficiently suppressed.

To simulate the real situation encountered in field measurements a small electric fan was used to produce continuous gas flow through the resonator and filters.

The light pulses for the excitation of the resonances were produced by a $\mathrm{XeCl}$ excimer laser (Lambda Physik LPX 105i). The average output power was $\approx 75 \mathrm{~mJ}$, and the pulse duration $\approx 20 \mathrm{~ns}$. As the beam quality was poor, only a fraction of the total pulse energy $(\approx 8 \mathrm{~mJ})$ passed through the beam-forming optical setup.

The PA and optical signals were fed into a digital oscilloscope (LeCroy 9400A), where they were digitized and averaged synchronously using the trigger signal of the laser control unit. The averaged data were transfered to a PC for FT and resonance profile fitting. Naphthalene vapor was chosen as the absorbing gas due to the excellent overlap of one of its absorption lines with the $308 \mathrm{~nm}$ emission line of the $\mathrm{XeCl}$ excimer [6].

As a first step the environmental noise was checked in the laboratory. The stationary 
a)

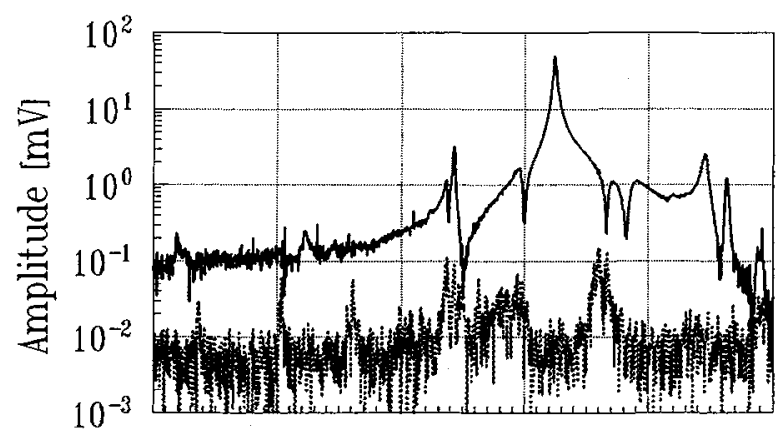

b)

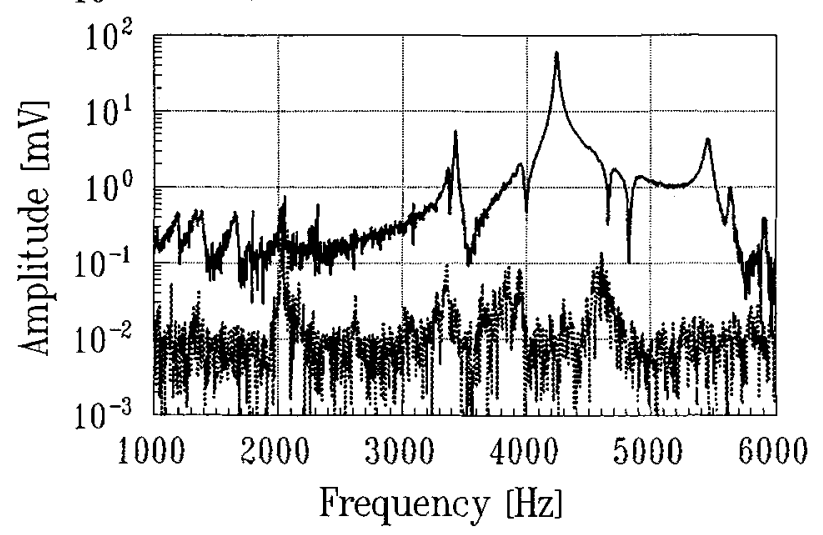

Figure 2: a) Spectra of PA signal and noise in the open resonator; b) spectra of PA signal and noise in the closed resonator (with windows)

background noise level was $\approx 60 \mathrm{~dB}$, while pulse noise signals due to excimer pulses, the door opening and closing, talking, etc., may have exceeded $75 \mathrm{~dB}$. The five-pole acoustic filters efficiently suppressed this noise, which was further reduced by the bandwidth-limited microphonepreamplifier response. The noise inside the resonator was measured with and without excimer laser pulses passing through the resonator, signal averaging, and air flow, separately. The broadband noise amplitudes were determined from the recorded data by calculating the standard deviation of the 4096 data points, while the spectral distribution was determined by Fourier transformation.

The noise figures were impressive. The acoustic and electric filter combination reduced the broadband noise by $50-55 \mathrm{~dB}$. This level was further reduced by $\approx 18 \mathrm{~dB}$ when 100 pulses were time averaged. Although the broadband noise increased considerably when the excimer laser and the air flow were switched on, the spectrum showed no significant noise production in the frequency range of the first radial resonance.

The spectrum of the PA signal and noise is shown in Fig. 2a. The signal spectrum shows only one strong peak around $4.2 \mathrm{kHz}$, which is the frequency of the first radial resonance of the resonator, while the other resonances of the cell and the Helmholtz resonances of the acoustic filters are efficiently suppressed. The spectra of the acoustic noise background in Fig. 2a,b are almost flat. The peaks produced by the flow were far away from the main resonance peak. Thus only a slight increase of $\approx 20 \%$ was observed around the resonance frequency. In this range the $\mathrm{S} / \mathrm{N}$ ratio was about $76 \mathrm{~dB}$.

To test the efficiency of the acoustic filters and the influence of windows mounted at 
both ends of the acoustic filters, additional spectra were measured showing a window signal below $2.5 \mathrm{kHz}$ (Fig. 2b). Comparing the two figures reveals two important results: first, the acoustic filters suppress the outside noise very efficiently, and second, the window signal does not interfere with the spectral range of the first radial mode if the windows are mounted at the ends of the acoustic filters.

\section{Conclusions}

The main results of this investigation can be summarized as follows:

- Pulsed laser excitation of a resonant PA resonator combined with synchronized averaging of the $\mathrm{PA}$ responses provides excellent $\mathrm{S} / \mathrm{N}$ ratios by suppressing the noise very efficiently.

- The required outside noise suppression can also be achieved with an open system by fitting acoustic filters at the openings of the resonator.

- In order to suppress the strong window signal, if windows are used they should be mounted at the ends of the acoustic filters.

- As the entire profile of the PA resonance signal is determined, a slow drift of the resonance frequency plays no role in these measurements. Thus, high-Q resonators can be used in this case.

- Flow-through operation is possible without serious degradation of the $\mathrm{S} / \mathrm{N}$ ratio.

- Background signals produced by light absorbed at the surfaces of the optical elements and the PA resonator's inner surface may reduce the dynamic range of the measurement considerably. Therefore it is useful to measure the background separately using a second beam tuned outside the absorption band of the species to be investigated.

\section{Acknowledgements}

This work was performed during a stay of A.M. in Heidelberg made possible by a Human Capital and Mobility fellowship granted by the European Union.

\section{References}

[1] Miklós A. and Lörincz A., Appl. Phys. B 48 (1989) 213.

[2] Angeli G. Z., Rev. Sci. Instrum. 62/63 (1991) 810.

[3] Carrer I., Fiorina L. and Zanzottera E., in Photothermal and Photoacoustic Phenomena $I I I$, Bićanić D. Ed. (Springer Ser. Opt. Sci. 69, Springer, Heidelberg, 1992) p. 568.

[4] Fiedler M. and Hess P., in Photoacoustic and Photothermal Phenomena, Murphy J. C., Maclachlan Spicer J. W. and Aamodt L. Eds. (Springer Ser. Opt. Sci. 62, Springer, Berlin, 1990) p. 344.

[5] Morse P. M. and Ingard K. U., Theoretical Acoustics (Princeton University Press, Princeton, 1968) pp. 490-492.

[6] Neuroth R., Dorn H.-P. and Platt U., J. Atmosph. Chem. 12 (1991) 287. 